\title{
EFICÁCIA DE HERBICIDAS EM DIFERENTES QUANTIDADES DE PALHA DE CANA-DE-AÇÚCAR NO CONTROLE DE IPOMOEA GRANDIFOLIA $\left(^{(1)}\right.$
}

\author{
PATRICIA ANDREA MONQUERO $\left({ }^{2 *}\right)$; LUCAS RIOS DO AMARAL $\left({ }^{3}\right)$; ANDRÉIA CRISTINA DA SILVA $\left({ }^{4}\right)$ \\ DENIS PAQUIER BINHA $\left({ }^{3}\right)$; PAULO VINICIUS DA SILVA $\left({ }^{3}\right)$
}

\begin{abstract}
RESUMO
Este trabalho foi realizado com o objetivo de estudar o efeito de diferentes quantidades de palha de cana-de-açúcar, colhida sem queima prévia do canavial, na eficácia de alguns herbicidas no controle de Ipomoea grandifolia. O experimento foi desenvolvido em campo no Centro de Ciências Agrárias/UFSCar, em Araras (SP). Os tratamentos consistiram dos herbicidas ametryn + trifloxysulfuron-sodium $(1463+37 \mathrm{~g}$ $\left.\mathrm{ha}^{-1}\right)$, imazapic $\left(84 \mathrm{~g} \mathrm{ha}^{-1}\right)$, imazapyr $\left(200 \mathrm{~g} \mathrm{ha}^{-1}\right)$, ${ }^{1}$ diuron + hexazinone $\left(1170+330 \mathrm{~g} \mathrm{ha}^{-1}\right) \mathrm{e}^{2}$ diuron + hexazinone $\left(1330+160 \mathrm{~g} \mathrm{ha}^{-1}\right)$ aplicados em pré-emergência de I. grandifolia e de cinco quantidades de palha de cana-de-açúcar $\left(0,5,10,15\right.$ e $\left.20 \mathrm{t} \mathrm{ha}^{-1}\right)$. A eficácia de controle das plantas daninhas foi avaliada aos $7,14,21$ e 28 dias após a aplicação dos tratamentos (DAT). Com a adição de palha (15 e $20 \mathrm{t} \mathrm{ha}^{-1}$ ) ao solo foi reduzida a população de I. grandifolia. À medida que a quantidade de palha foi aumentada, reduziu-se a eficácia dos herbicidas; com $15 \mathrm{t} \mathrm{ha}^{-1}$ de palha, o controle foi considerado insatisfatório para todos os tratamentos utilizados. Com $20 \mathrm{t} \mathrm{ha}^{-1}$ nenhum herbicida foi eficaz, entretanto, a palha foi eficiente na supressão desta espécie, verificando-se que o número de plantas emergidas foi menor mesmo na testemunha.
\end{abstract}

Palavras-chave: Saccharum spp, cobertura morta, amendoim bravo, controle químico.

\section{ABSTRACT \\ EFFECTIVENESS OF HERBICIDES THROUGH DIFFERENT AMOUNTS OF SUGAR-CANE STRAW ON THE IPOMOEA GRANDIFOLIA CONTROL}

This work had as objective studies the effect of different amounts of sugarcane straw picked without burns about effectiveness of some herbicides in the I. grandifolia control. The experiment was conduced in field in the Centro de Ciências Agrárias/UFSCar, in Araras, SP. The treatments consisted of herbicides herbicidas ametryn + trifloxysulfuron-sodium $\left(1463+37 \mathrm{~g} \mathrm{ha}^{-1}\right)$, imazapic $\left(84 \mathrm{~g} \mathrm{ha}^{-1}\right)$, imazapyr $(200 \mathrm{~g}$ $\left.\mathrm{ha}^{-1}\right),{ }^{1}$ diuron + hexazinone $\left(1170+330 \mathrm{~g} \mathrm{ha}^{-1}\right)$ and ${ }^{2}$ diuron + hexazinone $\left(1330+160 \mathrm{~g} \mathrm{ha}^{-1}\right)$ applied in pre emergence of Ipomoea grandifolia and on five growing amounts of sugarcane straw $(0,5,10,15 \mathrm{e} 20 \mathrm{t}$ $\left.\mathrm{ha}^{-1}\right)$. The effectiveness of control of weeds was evaluated to the 7, 14, 21 and 28 days after application of treatments (DAT).The straw reduced the population of I. grandifolia even in the treatments without herbicide use. The measure that increased the amount of straw there was reduction in the effectiveness of the herbicides and with $15 \mathrm{t} \mathrm{ha}^{-1}$ of straw the control was considered unsatisfactory for all of the treatments. With $20 \mathrm{t} \mathrm{ha}^{-1}$ the control was null for all the treatments, however, was observed that in this case, the own straw was efficient in the suppression of these weed, since the number of emerged plants was smaller, even in the treatment without herbicides.

Key words: Saccharum spp., mulching, chemical control.

( $\left.{ }^{1}\right)$ Recebido para publicação em 19 de setembro de 2006 e aceito em 6 de janeiro de 2009.

$\left(^{2}\right)$ Departamento de Recursos Naturais e Proteção Ambiental, Centro de Ciências Agrárias (UFSCar), Rodovia Anhanguera, km 174 - Araras (SP). E-mail: pamonque@cca.ufscar.br (*) Autora correspondente.

$\left(^{3}\right)$ Alunos de graduação do Centro de Ciências Agrárias/UFSCar.

( $\left.{ }^{4}\right)$ Agência Paulista de Tecnologia dos Agronegócios (APTA), Pólo Regional da Alta Sorocabana. E-mail: andreiacs@apta.sp.gov.br. 


\section{INTRODUÇÃO}

A colheita mecanizada da cana-de-açúcar está cada vez mais presente nos sistemas de produção no Brasil. Nesse sistema, sem queima, as folhas, bainhas, ponteiros, além de quantidade variável de pedaços de colmo são cortados, triturados e lançados sobre a superfície do solo, formando uma cobertura de resíduo vegetal (mulch) denominada palha ou palhada. A quantidade de palhada em canaviais colhidos sem queima é variável de 10 a $30 \mathrm{t} \mathrm{ha}^{-1}$, dependendo da cultivar empregada (TRIVELIN et al., 1996).

No caso de controle das plantas daninhas, em estudos realizados por diversos autores verificou-se que em algumas espécies predominantes na cultura de cana-de-açúcar há desenvolvimento diferenciado em função da quantidade de palha depositada no solo (Medina Melendez, 1990, Velini et al., 2000). Devido à cobertura morta são ocasionadas mudanças químicas, físicas e biológicas no solo, podendo provocar seleção da comunidade infestante, suprimindo-se a infestação de plantas daninhas normalmente consideradas importantes nos canaviais. RoDRIGUEs et al. (2000) constataram que em área com a presença de palha de cana-de-açúcar sobre o solo, houve redução em até $22 \%$ na germinação de Panicum maximum, $26 \%$ na de Brachiaria plantaginea, 49\% em Digitaria horizontalis, 83\% em Amaranthus hybridus e 90\% em B. decumbens. No entanto, o mesmo não é verificado com Euphorbia heterophylla e Ipomoea grandifolia, consideradas espécies-problema no sistema cana-crua (MARTINs et al., 1999; Rossi et al., 2006a; 2006b). A manutenção da palha na superfície do solo pode também dificultar a eficácia dos herbicidas aplicados em pré-emergência, pois neste sistema o transporte do herbicida até a superfície do solo é realizado pela água da chuva ou irrigação (MAciel e Velini, 2005; SimONi et al., 2006).

Segundo Freitas et al. (2004), em áreas de canade-açúcar o controle das plantas daninhas é realizado, principalmente, com herbicidas. Entre as diversas opções de herbicidas registrados para a cultura da cana-de-açúcar, têm-se os herbicidas inibidores da acetolactato sintase (ALS) como o imazapic, imazapyr, trifloxysulfuron-sodium (em mistura com ametryn), halosulfuron e flazasulfuron e os herbicidas inibidores da fotossíntese como o diuron isoladamente ou em mistura com hexazinone, ametryn, metribuzin e tebutiuron. A ação da maioria destes herbicidas é em pré e pós-emergência inicial, sendo recomendada no controle de gramíneas, folhas largas e perenes de difícil controle; pode ocorrer efeito residual nos solos superior a cem dias (Procópio et al., 2004).

Diversos autores têm realizado pesquisas quanto à lixiviação e ação dos herbicidas no sistema de cana crua. Segundo CAvenAghi et al. (2006a), com o aumento da quantidade de palha de cana sobre o solo, ocorre diminuição da quantidade do imazapic lixiviado pela chuva, principalmente em quantidades de $20 \mathrm{t} \mathrm{ha}^{-1}$. O mesmo resultado foi verificado com amicarbazone (CAVENAGHI et al., 2006b).

Na presença de até $10 \mathrm{t} \mathrm{ha}{ }^{-1}$ de palha de canade-açúcar, o controle das plantas daninhas Senna obtusifolia, I. nil, I. hederifolia e I. grandifolia, foi satisfatório quando aplicado o herbicida ametryn + trifloxysulfuronsodium. Com mais quantidades de palha foi reduzido a ação desta mistura (GravenA et al., 2004).

Com o diuron + hexazinone aplicado sobre quantidade de palha de $10 \mathrm{t} \mathrm{ha}^{-1}$, foi promovido controle de $85 \%$ de I. grandifolia, tanto em pré como em pós-emergência verificando-se menos controle de E. heterophylla (CORRÊA et al., 2006).

Objetivou-se com este trabalho avaliar a eficácia de três herbicidas inibidores da acetolactato sintase (ALS) e dois herbicidas inibidores da fotossíntese, utilizados na agroindústria canavieira para o controle de I. grandifolia, quando aplicados sobre diferentes quantidades de palha.

\section{MATERIAL E MÉTODOS}

O experimento foi realizado no município de Araras (SP), localizado a $22^{\circ} 18^{\prime}$ de latitude Sul e $47^{\circ} 18^{\prime}$ de longitude Oeste, entre 25/5/2006 e 30/6/2006. O clima, pela classificação de Koppen, é do tipo Cwa, mesotérmico com verões quentes e úmidos e invernos secos. Na análise química do solo (Latossolo VermelhoEscuro Distrófico), revelou-se $\mathrm{pH}$ em $\mathrm{CaCl}_{2}$ de 5,3; $22 \mathrm{~g}$ $\mathrm{dm}^{-3}$ de matéria orgânica; $12 \mathrm{mg} \mathrm{dm}^{-3}$ de $\mathrm{P}$ resina, $\mathrm{V}$ de $63 \%$, e teores de $\mathrm{K}, \mathrm{Ca}, \mathrm{Mg}, \mathrm{H}+\mathrm{Al}, \mathrm{SB}$ e CTC de 2,3; 28 ; 11; $24 ; 41,3$ e $65,3 \mathrm{mmol}_{\mathrm{c}} \mathrm{dm}^{-3}$. Foram monitoradas a temperatura média e a precipitação pluvial referentes ao período de desenvolvimento do experimento (Tabela 1).

A espécie I. grandifolia foi semeada em parcelas de $3 \mathrm{~m}^{2}$ com a finalidade de se obter 100 plantas por unidade experimental. As parcelas eram constituídas de cinco linhas de $3 \mathrm{~m}$ de comprimento e espaçadas de $0,20 \mathrm{~m}$, sendo considerada como bordadura uma linha de cada lado e $0,30 \mathrm{~m}$ em cada extremidade da parcela.

Após a semeadura foram colocadas sobre o solo quantidades de palha de cana-de-açúcar equivalentes a $0,5,10,15$ e $20 \mathrm{tha}^{-1}$. O delineamento experimental foi o de blocos ao acaso, em esquema fatorial $5 \times 5$, sendo avaliados cinco herbicidas, cinco níveis de palha e quatro repetições. Na área escolhida para instalação do experimento não havia infestação natural dessa espécie. 
Tabela 1. Dados climatológicos da região de Araras (SP), durante o experimento, entre 25/5/2006 e 30/6/2006

\begin{tabular}{|c|c|c|c|c|}
\hline \multirow{2}{*}{ Semanas após aplicação dos tratamentos } & \multicolumn{3}{|c|}{ Temperatura } & \multirow{2}{*}{ Chuva } \\
\hline & $\mathrm{T} \max$ & $\mathrm{T} \min$ & $\mathrm{T}$ med & \\
\hline & \multicolumn{3}{|c|}{$+{ }^{\circ} \mathrm{C}$} & $\mathrm{mm}$ \\
\hline $1 .^{\mathrm{a}}$ & 26,0 & 12,3 & 19,2 & - \\
\hline $2 .^{a}$ & 22,5 & 9,8 & 16,2 & 8,0 \\
\hline $3 .^{\mathrm{a}}$ & 26,8 & 10,8 & 18,8 & - \\
\hline $4 .^{\mathrm{a}}$ & 26,9 & 11,0 & 19,0 & 1,2 \\
\hline
\end{tabular}

Os tratamentos constaram dos herbicidas ametryn + trifloxysulfuron-sodium $\left(1463+37 \mathrm{~g} \mathrm{ha}^{-}\right.$ $\left.{ }^{1}\right)$, imazapic $\left(84 \mathrm{~g} \mathrm{ha}^{-1}\right)$, imazapyr $\left(200 \mathrm{~g} \mathrm{ha}^{-1}\right),{ }^{1}$ diuron + hexazinone $\left(1170+330 \mathrm{~g} \mathrm{ha}^{-1}\right) \mathrm{e}^{2}$ diuron + hexazinone $\left(1330+160 \mathrm{~g} \mathrm{ha}^{-1}\right)$ aplicados, em préemergência, em 28/5/2006, aos dois dias após a semeadura. Os herbicidas foram aplicados com pulverizador costal pressurizado por $\mathrm{CO}_{2}$, à pressão constante de $2,5 \mathrm{kgf} \mathrm{cm}^{2}$, com barra de aplicação provida de quatro bicos com pontas de pulverização do tipo leque 110.03 e consumo de calda equivalente a $200 \mathrm{~L} \mathrm{ha}^{-1}$. No inicio da aplicação, a umidade relativa do ar era de $60 \%$, temperatura de $28{ }^{\circ} \mathrm{C}$, e velocidade do vento de $4,0 \mathrm{~km} \mathrm{~h}^{-1}$. Após 24 horas, foi simulada uma chuva de $14 \mathrm{~mm}$. Esta simulação foi feita a cada dois dias após a aplicação dos herbicidas, até o fim do experimento, utilizando-se um sistema de irrigação por aspersão.

O controle das plantas daninhas foi avaliado aos $7,14,21$ e 28 dias após a aplicação dos tratamentos (DAT), por meio de uma escala percentual de notas, em que 0 (zero) correspondeu a nenhuma injúria na planta e 100 (cem) à morte das plantas, segundo a escala da Alam (1974). Na última avaliação, a palha foi retirada cuidadosamente das parcelas e contado o número de plantas emergidas de I. grandifolia.

Os resultados foram submetidos à análise de variância. A comparação entre as médias do efeito dos herbicidas dentro de cada quantidade de palha foi realizada empregando-se o teste de Tukey a 5\% de probabilidade. Para o efeito de época de avaliação e de quantidades de palha foi empregada a análise de regressão.

\section{RESULTADOS E DISCUSSÃO}

Houve interação significativa entre os diferentes herbicidas no controle de I. grandifolia. O desdobramento da interação aos 28 DAT é apresentado na tabela 2 .

Observou-se diferença significativa na eficácia dos herbicidas nos diferentes níveis de palha. Os herbicidas imazapic, imazapyr, ametryn + trifloxysulfuron-sodium $\mathrm{e}^{1}$ diuron + hexazinone foram interceptados quando foram depositados sobre o solo quantidades de palha iguais ou superiores a $15 \mathrm{t} \mathrm{ha}^{-1}$. Todavia, a mistura ${ }^{2}$ diuron + hexazinone foi a mais retida pela palha, havendo redução da eficácia a partir de $10 \mathrm{t} \mathrm{ha}^{-1}$ de palha.

Tabela 2. Controle percentual de I. grandifolia em função de diferentes herbicidas e quantidades de palha, avaliado aos 28 dias após a aplicação. Araras, 2006

\begin{tabular}{|c|c|c|c|c|c|}
\hline \multirow{2}{*}{ Herbicidas } & \multicolumn{5}{|c|}{ Palha de cana-de-açúcar $\left(\mathrm{t} \mathrm{ha}^{-1}\right)$} \\
\hline & 0 & 5 & 10 & 15 & 20 \\
\hline & \multicolumn{5}{|c|}{$\mathrm{C}_{\mathrm{L}} \%$} \\
\hline Imazapyr & $90,42 \mathrm{~b}$ & $87,50 \mathrm{~b}$ & $87,50 \mathrm{c}$ & $60,00 \mathrm{a}$ & $0,00 \mathrm{a}$ \\
\hline Imazapic & $90,00 \mathrm{~b}$ & $90,00 \mathrm{ab}$ & $85,00 \mathrm{c}$ & $52,50 \mathrm{a}$ & $0,00 \mathrm{a}$ \\
\hline Ametryn + trifloxysulfuron-sodium & 98,75 a & 91,25 a & $92,50 \mathrm{~b}$ & $60,00 \mathrm{a}$ & $0,00 \mathrm{a}$ \\
\hline${ }^{1}$ Diuron + hexazinone & $100,00 \mathrm{a}$ & 97,50 a & $96,25 \mathrm{a}$ & 58,75 a & $0,00 \mathrm{a}$ \\
\hline${ }^{2}$ Diuron + hexazinone & $100,00 \mathrm{a}$ & $91,25 \mathrm{a}$ & $22,50 \mathrm{~d}$ & $0,00 \mathrm{~b}$ & $0,00 \mathrm{a}$ \\
\hline CV $(\%)$ & 2,62 & 3,37 & 2,04 & 10,25 & 10,21 \\
\hline DMS (5\%) & 4,81 & 5,93 & 2,96 & 8,89 & 1,35 \\
\hline
\end{tabular}

Médias seguidas da mesma letra nas colunas não diferem significativamente entre si pelo Teste de Tukey a 5\% de probabilidade. 
Quanto aos herbicidas, dentro de cada nível de palha, verificou-se que, com $10 \mathrm{t} \mathrm{ha}^{-1}$ de palha houve diferença entre os herbicidas, sendo que ${ }^{2}$ diuron + hexazinone teve o pior desempenho no controle da espécie daninha $(22,50 \%)$. No nível de $15 \mathrm{t} \mathrm{ha}^{-1}$ de palha o pior desempenho foi obtido pela mistura ${ }^{2}$ diuron + hexazinone, seguido nominalmente pelo imazapic, ${ }^{1}$ diuron+hexazinone, imazazpyr e ametryn + trifloxysulfuron-sodium.Com $20 \mathrm{tha}^{-1}$ o controle foi nulo para todos os herbicidas estudados.

Na figura 1, constata-se o efeito dos herbicidas no controle de I. grandifolia quando aplicados em parcelas sem a cobertura de palha de cana-de-açúcar. As misturas com as duas doses de diuron + hexazinone tiveram um efeito mais rápido sobre as plantas daninhas, aproximando-se de $100 \%$ de controle aos 14 DAT. A mistura ametryn + trifloxysulfuron-sodium também foi eficaz no controle desta espécie, principalmente após 21 DAT. Com os herbicidas imazapic e imazapyr foi obtido controle lento ao longo do tempo. Segundo Rodrigues e Almeida (2005), as plantas afetadas pelos herbicidas inibidores da acetolactato sintase são levadas lentamente à morte, mas já poucas horas após o tratamento o crescimento é paralisado, com inibição da divisão celular.

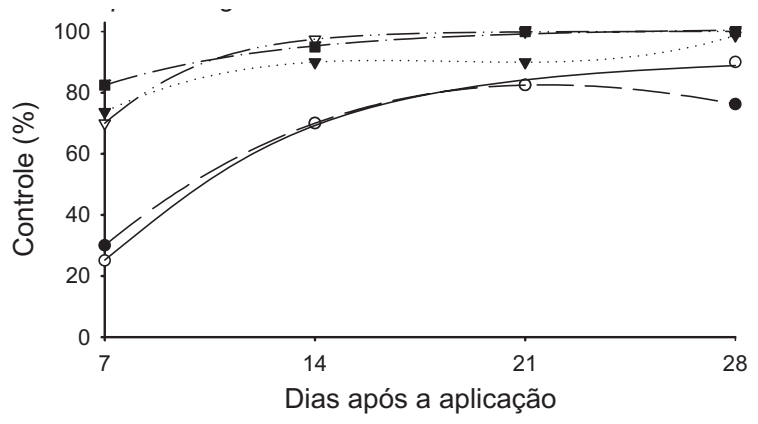

\footnotetext{
$\rightarrow-\operatorname{Imazapyr}\left(\mathrm{Y}=-46,25+13,8988 \mathrm{x}-0,4592 \mathrm{x}^{2}+0,0043 \mathrm{x}^{3}\right) \mathrm{R}^{2}=1,0$

- $\quad$ Imazapic $\left(Y=92,6951 /\left(1+(x / 9,7369)^{-2,9853}\right)\right) R^{2}=0,998$

- A Ametryn + Trifloxysulfuron sodium $\left(Y=16,25+12,3512 x-0,6760 x^{2}+0,0121 x^{3}\right) R^{2}=1,0$

$\nabla{ }^{1}$ Diuron + Hexazinone $(Y=100,0813 / 1+\exp (-(x-4,8816) / 2,5083)) R^{2}=0,999$

- ${ }^{2}$ Diuron + Hexazinone $\left(Y=(100,9912-60,6531) /(1+0,0001 x)^{0,5}\right) R^{2}=0,995$
}

Figura 1. Eficácia dos herbicidas imazapyr $\left(200 \mathrm{~g} \mathrm{ha}^{-1}\right)$, imazapic $\left(84 \mathrm{~g} \mathrm{ha}^{-1}\right)$, ametryn + trifloxysulfuron-sodium $\left(1463+37 \mathrm{~g} \mathrm{ha}^{-1}\right),{ }^{1}$ diuron + hexazinone $(1170+330 \mathrm{~g}$ $\left.\mathrm{ha}^{-1}\right) \mathrm{e}^{2}$ diuron + hexazinone $\left(1330+160 \mathrm{~g} \mathrm{ha}^{-1}\right)$ aplicados em pré-emergência no controle de I. grandifolia, avaliada aos 7, 14, 21 e 28 DAT nas parcelas sem palha de canade-açúcar. Araras (SP), 2006.

Na figura 2, ou seja, com $5 \mathrm{t} \mathrm{ha}^{-1}$ de palha, não foi observada grande diferença no controle dos herbicidas em relação ao tratamento sem a presença de palha. Aos 28 DAT todos os herbicidas foram eficientes, destacando-se as misturas ${ }^{1,2}$ diuron + hexazinone com controle de $100 \%$ de I. grandifolia.
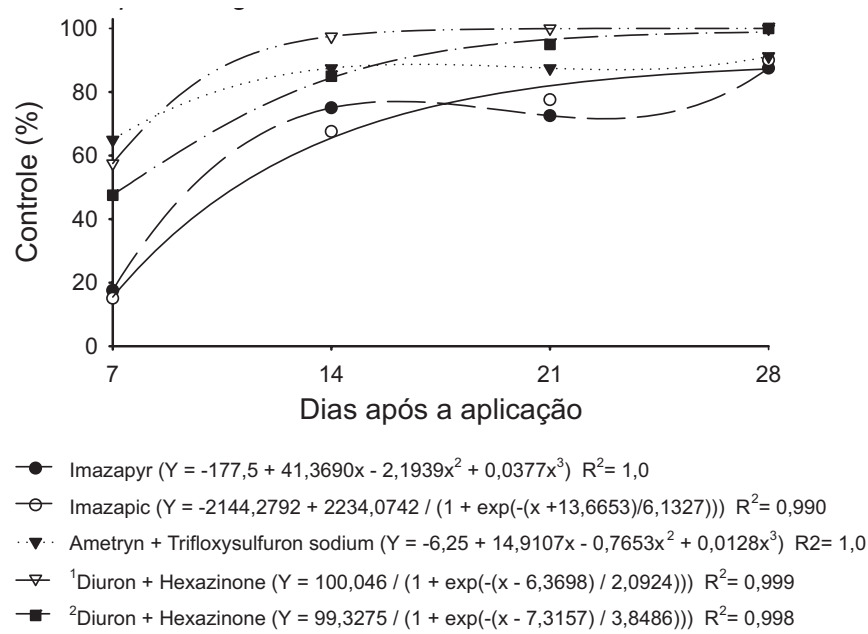

Figura 2. Eficácia dos herbicidas imazapyr (200 $\left.\mathrm{g} \mathrm{ha}^{-1}\right)$, imazapic $\left(84 \mathrm{~g} \mathrm{ha}^{-1}\right)$, ametryn + trifloxysulfuron-sodium $\left(1463+37 \mathrm{~g} \mathrm{ha}^{-1}\right),{ }^{1}$ diuron + hexazinone $(1170+330 \mathrm{~g}$ $\left.\mathrm{ha}^{-1}\right) \mathrm{e}^{2}$ diuron + hexazinone $\left(1330+160 \mathrm{~g} \mathrm{ha}^{-1}\right)$ aplicados em pré-emergência no controle de I. grandifolia, avaliada aos $7,14,21$ e 28 DAT nas parcelas com $5 \mathrm{t} \mathrm{ha}^{-1}$ palha de cana-de-açúcar. Araras (SP), 2006.

À medida que se aumentou a quantidade de palha de cana-de-açúcar nas parcelas, a eficácia dos herbicidas foi diminuindo. Quando se adicionaram ao solo $10 \mathrm{t} \mathrm{ha}^{-1}$, o controle foi satisfatório $(>80 \%$ de controle) com a aplicação de ametryn + trifloxysulfuronsodium, imazapic, imazapyr e ${ }^{1}$ diuron + hexazinone (Figura 3). Considerando que nesta formulação de diuron + hexazinone há concentração maior de hexazinone sugere-se que este ingrediente ativo foi mais eficaz em atravessar a palha. Herbicidas de baixa solubilidade como o diuron $\left(42 \mathrm{ppm}\right.$ a $\left.25^{\circ} \mathrm{C}\right)$, necessitam de maior teor água no solo para se movimentar e atuar, quando comparados ao hexazinone que possui alta solubilidade $(29.800 \mathrm{ppm}$ a $25^{\circ} \mathrm{C}$ ) (BOUCHARD et al., 1985).

Com $15 \mathrm{t} \mathrm{ha}^{-1}$ de palha, o controle foi ineficaz em todos os tratamentos. Entretanto, os maiores níveis de controle foram obtidos com imazapyr e ametryn + trifloxysulfuron-sodium, já que, com ambos, na ultima avaliação obtiveram-se $60 \%$ de controle (Figura 4 ). Com $20 \mathrm{t} \mathrm{ha}^{-1}$ de palha a porcentagem de controle de todos os herbicidas foi nula, entretanto, observou-se que, neste caso, a própria palha foi eficiente na supressão de I. grandifolia, já que o número de plantas emergidas foi menor à medida que se aumentou a quantidade de palha, mesmo na testemunha (Figura 5). No controle químico a camada de palha pode prejudicar a ação do herbicida aplicado. Foi constatado por Velini e Negrisoli (2000) que com 10 a $15 \mathrm{t} \mathrm{ha}^{-1}$ de palha podem ser interceptados aproximadamente $99,5 \%$ da calda aplicada. Em outro trabalho, cerca de $90 \%$ do imazaquin aplicado ficaram 
retidos na palha de Avena strigosa antes da irrigação e, após a irrigação, menos de $7 \%$, em média, ficaram na palha, e $20 \mathrm{~mm}$ de chuva foram suficientes para a lixiviação do produto da palha para o solo (RoDRIGUES et al., 2000). De forma semelhante, ForNARIOLLI (1997) verificou que $85 \%$ do atrazine ficaram retidos na palha e que uma chuva de $20 \mathrm{~mm}$ foi suficiente para o arraste de praticamente todo o produto da palha para o solo.

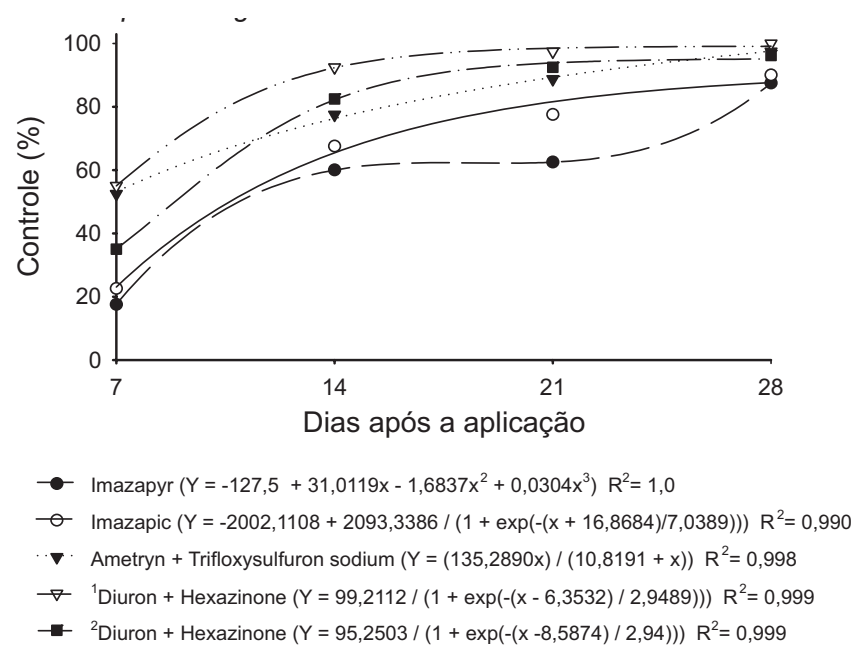

Figura 3. Eficácia dos herbicidas imazapyr $\left(200 \mathrm{~g} \mathrm{ha}^{-1}\right)$, imazapic $\left(84 \mathrm{~g} \mathrm{ha}^{-1}\right)$, ametryn + trifloxysulfuron-sodium $\left(1463+37 \mathrm{~g} \mathrm{ha}^{-1}\right),{ }^{1}$ diuron + hexazinone $(1170+330 \mathrm{~g}$ $\left.\mathrm{ha}^{-1}\right) \mathrm{e}^{2}$ diuron + hexazinone $\left(1330+160 \mathrm{~g} \mathrm{ha}^{-1}\right)$ aplicados em pré-emergência no controle de I. grandifolia, avaliada aos $7,14,21$ e 28 DAT nas parcelas com $10 \mathrm{t} \mathrm{ha}^{-1}$ palha de cana-de-açúcar. Araras (SP), 2006.

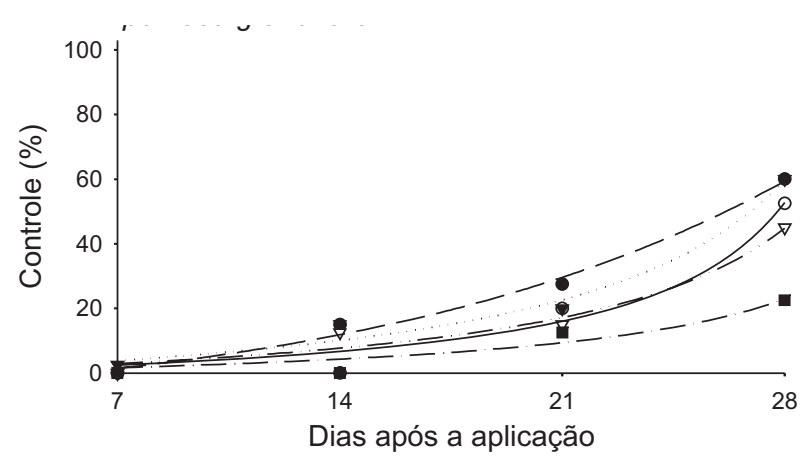

- Imazapyr $(\mathrm{Y}=-12,9774+1386,8875 /(1+\exp (-(\mathrm{X}-64,7312) / 12,6662))) \mathrm{R}^{2}=0,991$

- $\quad$ Imazapic $(\mathrm{Y}=(0,2732 \mathrm{x}) /(1-0,0305 \mathrm{x})) \mathrm{R}^{2}=0,959$

V Ametryn + Trifloxysulfuron sodium $(Y=(0,4330 x) /(1-0,0285 x)) R^{2}=0,983$

$\nabla{ }^{1}$ Diuron + Hexazinone $(Y=(0,3312 x) /(1-0,0283 x)) R^{2}=0,967$

- ${ }^{2}$ Diuron + Hexazinone $(Y=(0,1883 x) /(1-0,0275 x)) R^{2}=0,897$

Figura 4. Eficácia dos herbicidas imazapyr (200 $\left.\mathrm{g} \mathrm{ha}^{-1}\right)$, imazapic $\left(84 \mathrm{~g} \mathrm{ha}^{-1}\right)$, ametryn + trifloxysulfuron-sodium $\left(1463+37 \mathrm{~g} \mathrm{ha}^{-1}\right),{ }^{1}$ diuron + hexazinone $(1170+330 \mathrm{~g}$ ha $\left.^{-1}\right) \mathrm{e}^{2}$ diuron + hexazinone $\left(1330+160 \mathrm{~g} \mathrm{ha}^{-1}\right)$ aplicados em pré-emergência no controle de I. grandifolia, avaliada aos $7,14,21$ e 28 DAT nas parcelas com $15 \mathrm{t} \mathrm{ha}^{-1}$ palha de cana-de-açúcar. Araras (SP), 2006.

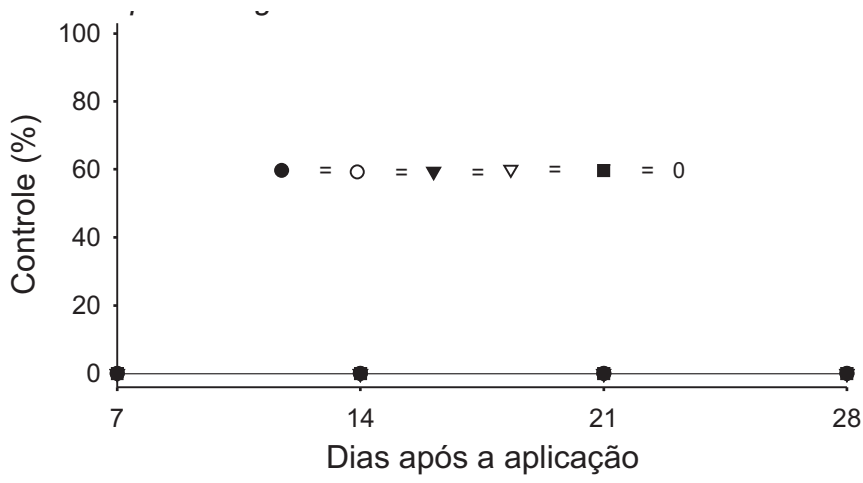

Figura 5. Eficácia dos herbicidas imazapyr (200 $\left.\mathrm{g} \mathrm{ha}^{-1}\right)$, imazapic $\left(84 \mathrm{~g} \mathrm{ha}^{-1}\right)$, ametryn + trifloxysulfuron-sodium $\left(1463+37 \mathrm{~g} \mathrm{ha}^{-1}\right),{ }^{1}$ diuron + hexazinone $(1170+330 \mathrm{~g}$ $\left.\mathrm{ha}^{-1}\right) \mathrm{e}^{2}$ diuron + hexazinone $\left(1330+160 \mathrm{~g} \mathrm{ha}^{-1}\right)$ aplicados em pré-emergência no controle de I. grandifolia, avaliada aos $7,14,21$ e 28 DAT nas parcelas com $20 \mathrm{t} \mathrm{ha}^{-1}$ palha de cana-de-açúcar. Araras (SP), 2006.

Observou-se que, com cobertura do solo com 15 e $20 \mathrm{t} \mathrm{ha}^{-1}$ de palha, tanto nas parcelas com e sem aplicação de herbicidas, proporcionou-se redução na emergência de I. grandifolia (Figura 6). Portanto, na presença de 15 ou 20 t ha ${ }^{-1}$ de palha de cana-de-açúcar, o uso de herbicidas para o controle de I. grandifolia pode ser desnecessário.

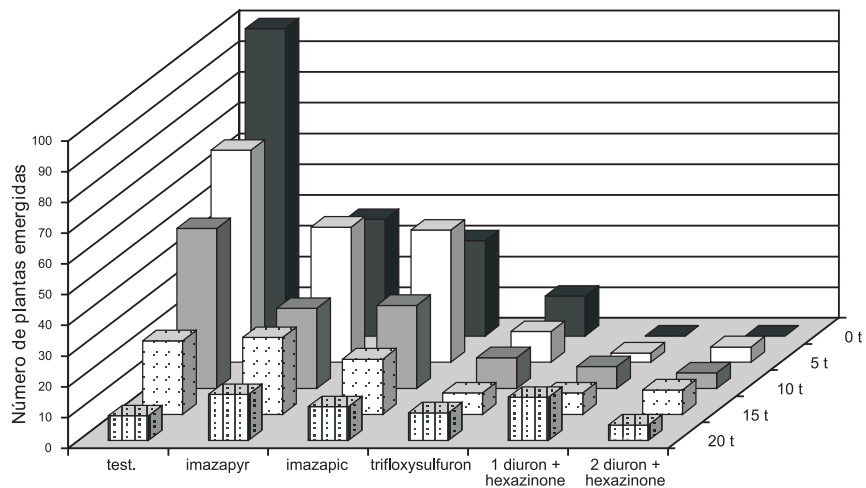

Figura 6. Número de plantas de I. grandifolia emergidas em diferentes quantidades de palha de cana-de-açúcar e herbicidas $(D M S=$ diferença mínima significativa: $\mathrm{P}<0,05)$. Araras (SP), 2006.

\section{CONCLUSÕES}

1. Com quantidades de palha de cana-deaçúcar maiores que $10 \mathrm{t} \mathrm{ha}^{-1}$ há retenção e, portanto, diminuição da eficácia dos herbicidas ametryn + trifloxysulfuron-sodium, imazapic, imazapyr e diuron + hexazinone aplicados em pré-emergência.

2. Na cobertura do solo com 15 e $20 \mathrm{t} \mathrm{ha}^{-1}$ de palha de cana-de-açúcar, tanto com quanto sem aplicação de herbicidas, há redução na emergência de I. grandifolia. 


\section{AGRADECIMENTOS}

À Fundação de Amparo à Pesquisa do Estado de São Paulo (FAPESP), que proporcionou condições para que esta pesquisa fosse realizada e aos técnicos Rubens Olívio e Ernesto Favetta que ajudaram no desenvolvimento do trabalho.

\section{REFERÊNCIAS}

BOUCHARD, D. C.; LAVY, T. L.; LAWSON, E. R. Mobility and persistence of hexazinone in a forest watershed. Journal of Environmental Quality, Bethesda, v.14, n.2, p.229-233, 1985.

CAVENAGHI, A. L.; VELINI, E.D.; CORREAA, M.R.; MEDEIROS, D.; DEGASPARI, N. Dinâmica do herbicida imazapic aplicado sobre a palha de cana-de-açúcar. In: CONGRESSO BRASILEIRO DA CIÊNCIA DAS PLANTAS DANINHAS, 25, 2006. Brasília, Resumos... Brasília: SBCPD; UNB; Embrapa Cerrados, 2006a. p. 360.

CAVENAGHI, A. L.; ROSSI, C.V.S.; NEGRISOLI, E.; COSTA, E.A.D; VELINI, E.D.; TOLETO, R.E.B. Performance do herbicida amicarbazone aplicado sobre a palha de cana-de-açúcar. In: CONGRESSO BRASILEIRO DA CIÊNCIA DAS PLANTAS DANINHAS, 25, Brasília, 2006b. Resumos... Brasília: SBCPD; UNB; Embrapa Cerrados, 2006. p. 330.

CORRÊA, M.R.; ROSSI, C.V.S.; NEGRISOLI, E.; SILVA, F.M.L.; VELINI, E.D.; PANINI, E.L.; OLIVEIRA, C.P. Eficiência do herbicida Diuron + Hexazinone no controle de plantas daninhas na cultura de cana-de-açúcar em aplicações em pré e pós-emergência. In: CONGRESSO BRASILEIRO DA CIÊNCIA DAS PLANTAS DANINHAS, 25, Brasília, 2006. Resumos... Brasília: SBCPD; UNB; Embrapa Cerrados, 2006. p. 364.

FORNARIOLLI, D.A. Influência da cobertura morta no comportamento do herbicida atrazine. 1997. 124p. Dissertação (Mestrado) - Universidade Estadual de Londrina, Londrina, PR.

FREITAS, S. P.; OLIVEIRA, A.R.; FREITAS, S.J.; SOARES, L.M.S. Controle químico de Rottboelia exaltata em cana-de-açúcar. Planta Daninha, Viçosa, v.22, n.3, p.461-466, 2004.

GRAVENA, R., RODRIGUES, J.P.R.G., SPINDOLA, W., PITELLI, R.A. e ALVES, P.L.C.A. Controle de plantas daninhas através da palha de cana-de-açúcar associada à mistura dos herbicidas trifloxysulfuron sodium + ametryn. Planta Daninha, Viçosa, v.22, n.3, p.419-427, 2004.

MACIEL, C.D.G.; VELINI, E.D. Simulação do caminhamento da água da chuva e herbicidas em palhadas utilizadas em sistemas de plantio direto. Planta Daninha, Viçosa, v.23, n.3, p.471-481, 2005.

MARTINS, D.; VELINI, E.D.; MARTINS C.C.; SOUZA, L.S. Emergência em campo de dicotiledôneas daninhas em solo coberto com palha de cana-de-açúcar. Planta Daninha,Viçosa, v.17, n.1, p.151-161, 1999.
MEDINA MELENDEZ, J. A. Efeito da cobertura do solo no controle de plantas daninhas na cultura do pepino (Cucumis sativus L.). 1990. 104 f. Dissertação (Mestrado) - Escola Superior de Agricultura "Luiz de Queiroz", Piracicaba, 1990.

PROCÓPIO, S. O.; SILVA, A. A.; VARGAS, L. Manejo e controle de plantas daninhas em cana-de-açúcar. In.: VARGAS, L.; ROMAN, E. S. (Eds.). Manual de manejo e controle de plantas daninhas. Bento Gonçalves: Embrapa Uva e Vinho, 2004. p.397-452.

RODRIGUES, B.N. \& F.S. ALMEIDA. Guia de herbicidas. 5.ed. Londrina: IAPAR, 2005. 591p.

RODRIGUES, B.N.; LIMA, J.; YADA, I.F.U.; ULBRICH, A.V.; FORNARIOLLI, D. Influência da cobertura morta na retenção do imazaquin em plantio direto de soja. Planta Daninha, Viçosa, v.18, n. 2, p. 231-239, 2000.

ROSSI, C.V.S. ; VELINI, E.D.; PIVETTA, J.P.; SILVA, F.M.L.; NEGRISOLI, E.; CORRÊA, M.R.; FOGANHOLI, L.A.P.; COSTA, A.G.F. Efeito da presença de palha de cana crua a germinação de plantas daninhas em época seca. In: CONGRESSO BRASILEIRO DA CIÊNCIA DAS PLANTAS DANINHAS, 25., 2006. Brasília, Resumos... Brasília: SBCPD; UNB; Embrapa Cerrados, 2006a. p. 326.

ROSSI, C.V.S. ; VELINI, E.D.; PIVETTA, J.P.; SILVA, F.M.L.; NEGRISOLI, E.; CORRÊA, M.R.; FOGANHOLI, L.A.P.; COSTA, A.G.F. Efeito da presença de palha de cana crua sobre a germinação de plantas daninhas em época úmida. In: CONGRESSO BRASILEIRO DA CIÊNCIA DAS PLANTAS DANINHAS, 25., 2006. Brasília, Resumos... Brasília: SBCPD; UNB; Embrapa Cerrados, 2006b. p. 346.

SIMONI, F.; VICTORIA FILHO, R.; SAN MARTIN, H.A.M.; SALVADOR, F.L.; ALVES, A.S.R.; BREMER, N. H. Influência da intensidade de chuva e da quantidade de palha de cana-deaçúcar sobre a eficácia de herbicidas aplicados em préemergência no controle de Cyperus rotundus. In: CONGRESSO BRASILEIRO DA CIÊNCIA DAS PLANTAS DANINHAS, 25., 2006. Brasília, Resumos... Brasília: SBCPD; UNB; Embrapa Cerrados, 2006. p. 365.

VELINI, E. D. et al. Efeito da palha da cana-de-açúcar sobre a germinação das principais espécies de plantas daninhas gramíneas desta cultura. In: CONGRESSO BRASILEIRO DA CIÊNCIA DAS PLANTAS DANINHAS, 22., 2000, Foz do Iguaçu. Resumos... Londrina: SBCPD, 2000. p. 15.

VELINI, E. D.; NEGRISOLI, E. Controle de plantas daninhas em cana crua. In: CONGRESSO BRASILEIRO DA CIÊNCIA DAS PLANTAS DANINHAS, 22., 2000, Foz do Iguaçu. Palestras... Foz do Iguaçu: SBCPD, 2000. p. 148-164.

TIMOSSI, P.C.; DURIGAN, J.C. Manejo de convolvuláceas em dois cultivares de soja semeada diretamente sob palha residual de cana crua. Planta Daninha,Viçosa, v.24, n.1, p.91-98, 2006.

TRIVELIN, P.C.O.; RODRIGUES, J.C.S.; VICTORIA, R.L.; REICHARDT, K. Utilização por soqueira de cana-de-açúcar de safra do nitrogênio da aquamônia-15N e uréia-15N aplicado ao solo em complemento a vinhaça. Pesquisa Agropecuária Brasileira, Brasília, v.31, n.1, p.89-99, 1996. 